\title{
As atitudes dos profissionais de saúde mental frente ao uso de álcool
}

\author{
Attitudes of mental health professionals towards alcohol use \\ Actitudes de los profesionales de la salud mental hacia el consumo de alcohol
}

Recebido: 01/07/2021 | Revisado: 09/07/2021 | Aceito: 12/07/2021 | Publicado: 22/07/2021

\author{
Amanda Assis de Almeida \\ ORCID: https://orcid.org/0000-0002-6322-041X \\ Universidade Estadual Paulista "Júlio de Mesquita Filho", Brasil \\ E-mail: amandaaassis_@hotmail.com \\ Thiago da Silva Domingos \\ ORCID: https://orcid.org/0000-0002-1421-7468 \\ Universidade Federal de São Paulo, Brasil \\ E-mail: t.domingos@unifesp.br \\ Guilherme Correa Barbosa \\ ORCID: https://orcid.org/0000-0002-7433-8237 \\ Universidade Estadual Paulista “Júlio de Mesquita Filho", Brasil \\ E-mail: g.barbosa@unesp.br
}

\begin{abstract}
Resumo
Objetivo: verificar associações entre o perfil sociodemográfico e as atitudes de profissionais de um serviço de referência em álcool de outras drogas, frente ao álcool, ao alcoolista e ao alcoolismo. Método: estudo transversal, realizado com 42 profissionais de um serviço especializado no cuidado ao usuário com transtornos decorrentes do uso de álcool e outras drogas. Para a coleta de dados aplicou-se um questionário sociodemográfico e a Escala de Atitudes Frente ao Álcool, Alcoolismo e Alcoolista. Utilizou-se modelos de regressão univariada e múltipla para verificar a associação entre os desfechos. Resultados: observou-se atitudes positivas nos domínios teóricos de trabalho e relação estabelecida junto ao usuário, e negativas em relação ao uso de álcool e sua etiologia. Não foram identificadas associações entre as variáveis sociodemográficas e as atitudes profissionais. Conclusão: as atitudes frente ao álcool, alcoolismo e alcoolista não estão associadas às variáveis sociodemográficas dos profissionais de saúde no contexto de um serviço especializado.
\end{abstract}

Palavras-chave: Atitude do pessoal de saúde; Transtornos relacionados ao uso de álcool; Serviços de saúde mental; Conhecimento; Redução do dano.

\begin{abstract}
Objective: to verify associations between the sociodemographic profile and the attitudes of professionals from a reference service on alcohol and other drugs, towards alcohol, alcoholics and alcoholism. Method: cross-sectional study carried out with 42 professionals from a service specialized in caring for users with disorders resulting from the use of alcohol and other drugs. For data collection, a sociodemographic questionnaire and the Scale of Attitudes towards Alcohol, Alcoholism and Alcoholism were applied. Univariate and multiple regression models were used to verify the association between outcomes. Results: positive attitudes were observed in the theoretical domains of work and the relationship established with the user, and negative attitudes towards alcohol use and its etiology. No associations were identified between sociodemographic variables and professional attitudes. Conclusion: attitudes towards alcohol, alcoholism and alcoholics are not associated with sociodemographic variables of health professionals in the context of a specialized service.
\end{abstract}

Keywords: Attitude of health personnel; Alcohol-related disorders; Mental health services; Knowledge; Harm reduction.

\section{Resumen}

Objetivo: verificar asociaciones entre el perfil sociodemográfico y las actitudes de los profesionales de un servicio de referencia en alcohol y otras drogas, hacia el alcohol, los alcohólicos y el alcoholismo. Método: estudio transversal realizado con 42 profesionales de un servicio especializado en atención a usuarios con trastornos derivados del consumo de alcohol y otras drogas. Para la recolección de datos se aplicó un cuestionario sociodemográfico y la Escala de Actitudes hacia el Alcohol, Alcoholismo y Alcoholismo. Se utilizaron modelos de regresión univariante y múltiple para verificar la asociación entre los resultados. Resultados: se observaron actitudes positivas en los dominios teóricos del trabajo y la relación establecida con el usuario, y actitudes negativas hacia el consumo de alcohol y su etiología. No se identificaron asociaciones entre variables sociodemográficas y actitudes profesionales. Conclusión: las actitudes hacia el alcohol, el alcoholismo y los alcohólicos no se asocian con variables sociodemográficas de los profesionales de la salud en el contexto de un servicio especializado. 
Palabras clave: Actitud del personal de salud; Trastornos relacionados con alcohol; Servicios de salud mental; Conocimiento; Reducción del daño.

\section{Introdução}

O uso de substâncias psicoativas existe em diferentes culturas caracterizado entre distintas funcionalidades, cenários e padrões de consumo (World Health Organization [WHO], 2018). Em se tratando de uma das substâncias mais consumidas em caráter nocivo e prejudicial no âmbito mundial, o consumo de álcool está associado a muitos problemas de saúde pública (Costa \& Paiva, 2016; Mangueira, Guimarães, Mangueira, Fernandes \& Lopes, 2015).

No contexto global, o consumo do álcool e os transtornos relacionados estão entre os mais prevalentes dos transtornos mentais e comportamentais, acometendo 8,6\% entre os homens e 1,7\% das mulheres (Carvalho, Heilig, Perez, Probst, Rehm, 2019). Observou-se, no Brasil, um discreto aumento na proporção de pessoas não abstinentes entre os anos de 2006 (48\%) e 2012 (50\%). Em 2006, 29\% afirmaram consumir cinco doses ou mais e, em 2012, esta proporção aumentou para 39\% (Mangueira et al., 2015). O número de pessoas que bebem pelo menos uma vez por semana cresceu de $42 \%$ para $53 \%$ no mesmo período. Além desses dados, o início do uso de álcool tem sido cada vez mais precoce (WHO, 2018; Mangueira et al., 2015) e a análise epidemiológica indica uma tendência para a diminuição da diferença entre os sexos, o que significa afirmar, aumento da prevalência entre pessoas do sexo feminino (Carvalho et al., 2019).

Nesse rumo, o consumo de álcool associa-se à alta Carga Global de Doenças, desenvolvendo condições de morbidade e mortalidade relacionadas ao efeito tóxico sobre diversos sistemas biológicos e ao desenvolvimento de dependência e intoxicação aguda (WHO, 2018; Carvalho et al., 2019). De certo modo, esse cenário está relacionado à baixa prevalência do tratamento, que na perspectiva global está em torno de $21,9 \%$, representando uma das menores taxas quando em comparação a qualquer outro transtorno mental. Os sistemas de saúde, o usuário e o profissional estão entre os fatores que explicam essa problemática (Carvalho et al., 2019).

A efetivação de políticas públicas como meio para o enfrentamento dos prejuízos gerados pelo abuso de álcool é fundamental e deve ser operacionalizada por ações intersetoriais que se estendam ao conjunto de fatores condicionantes e relacionados à saúde, com vistas a intervir nas diferentes dimensões que são inerentes à problemática do consumo de álcool, assegurando à pessoa o acesso equitativo a bens e serviços, inclusive a atenção à saúde (WHO, 2018; Costa \& Paiva, 2016).

No contexto das políticas de saúde mental, a Reforma Psiquiátrica Brasileira tem como um de seus objetivos desconstruir a exclusão sofrida pelas pessoas com transtornos mentais promovendo a reinserção social da atuação de equipes multiprofissionais na composição de um projeto de cuidado que atenda às diretrizes do Sistema Único de Saúde (SUS) e aos princípios sociocultural, político-jurídico, técnico-assistencial e epistêmico da reforma (Carvalho et al., 2019; Vargas, Bittencourt, Rocha \& Silva, 2014).

No influxo de todos esses elementos estão as atitudes dos profissionais, objetos de investigação desde a década de 1960. Os valores, as crenças e os sentimentos compreendem os componentes dessas atitudes e atuam como preditores de comportamentos ou de ações frente a uma determinada situação. Assim, no contexto da assistência à saúde, as atitudes estão de certa maneira associadas à produção do cuidado, em síntese, uma parte da intervenção oferecida aos usuários pelos profissionais de saúde evidencia suas predisposições (Ramírez \& Vargas, 2017; Pinho, Oliveira, Claro, Soares, Gonçalves \& Pereira, 2018).

Esses componentes quando caracterizados como traços latentes podem ser analisados por meio de instrumentos que possibilitam avaliar e compreender sua natureza, causas e consequências em diversos contextos. Essa análise proporciona uma abordagem educativa que potencialmente pode produzir transformações comportamentais e atitudinais nos trabalhadores para a qualificação do cuidado à pessoa com transtornos mentais decorrentes do uso de álcool (Ramírez \& Vargas, 2017). 
Investigações que analisam a influência das atitudes profissional têm sido realizadas com profissionais da equipe multiprofissional em distintos contextos assistenciais. Reconhece-se que as atitudes de profissionais refletem diretamente sobre a assistência prestada e sobre as boas práticas. Correlações e associações entre as características sociodemográficas, profissionais e ocupacionais dos trabalhadores da área da saúde são identificadas como possíveis fatores que modificam as atitudes (Pinho et al., 2018; Orsoliniet al., 2021; Deng et al., 2021; Vistorte et al., 2018; Richards, Broadus \& Yerdelman, 2021; Bezerra, Freitas \& Amendola, 2020).

Profissionais de saúde com atitudes negativas demonstra uma maior carga moralista, que por sua vez dificulta o reconhecimento dos problemas relacionados ao uso do álcool. Em contrapartida, as capacitações técnica e relacional oferecidas aos profissionais contribuem positivamente para mudanças em suas atitudes que, consequentemente, diminuem as repercussões provenientes de abordagens moralistas (Pinho et al., 2018; Orsoliniet al., 2021; Deng et al., 2021; Vistorte et al., 2018; Richards, Broadus \& Yerdelman, 2021; Bezerra, Freitas \& Amendola, 2020). Observa-se como lacuna estudos que analisem as atitudes dos profissionais da equipe de saúde que atua no contexto de serviços especializados.

Pelo exposto, pretende-se com essa pesquisa responder à seguinte pergunta: as características sociodemográficas dos trabalhadores de um serviço de referência em álcool e outras drogas exercem influências sobre as atitudes que esses trabalhadores apresentam frente ao álcool, ao alcoolista e ao alcoolismo? Diante desse questionamento, objetiva-se verificar associações entre o perfil sociodemográfico e as atitudes de profissionais de um serviço de referência em álcool de outras drogas, frente ao álcool, ao alcoolista e ao alcoolismo.

\section{Metodologia}

Trata-se de um estudo transversal conduzido em um serviço público de referência em álcool e outras drogas em um município do interior do estado de São Paulo (Polit \& Beck, 2018). Possui 40 leitos operacionalmente ativos e atende, regionalmente, os 68 municípios que estão circunscritos ao Departamento Regional de Saúde VI.

Participaram todos os 42 trabalhadores do referido serviço com formação na área da saúde, com mínimo de um ano de experiência no serviço, e que não estavam de férias ou de licença médica.

Os dados foram obtidos entre os meses de março e maio de 2018 por meio de entrevista individual com aplicação de um questionário para obtenção de dados sociodemográficos e da Escala de Atitudes Frente ao Álcool, Alcoolismo e Alcoolista (EAFAAA) para a identificação dos dados sobre as atitudes dos trabalhadores frente ao álcool, alcoolismo e alcoolista (Vargas \& Rocha, 2016; Vargas \& Luis, 2008).

A versão EAFAAA contém 50 afirmativas relacionadas aos quatro grupos de atitudes expressas pelos profissionais de saúde frente ao álcool, alcoolismo e alcoolista (Vargas \& Rocha, 2016; Vargas \& Luis, 2008). As afirmativas estão distribuídas em quatro fatores: Fator 1 - O trabalhar e o relacionar-se com o alcoolista, possui 21 itens; Fator 2 - As atitudes frente ao alcoolista, nove itens; Fator 3 - Atitudes frente ao alcoolismo, etiologia com 11 itens; e Fator 4 - As atitudes frente ao uso do álcool, com nove itens (Vargas \& Rocha, 2016; Vargas \& Luis, 2008).

A resposta para cada uma das afirmativas é organizada segundo uma escala Likert de cinco pontos (1 - discordo totalmente, 2 - discordo, 3 - indiferente, 4 - concordo e 5 - concordo totalmente). Dentre os itens, alguns possuem lógica invertida e devem ser considerados para o cálculo das atitudes. De uma maneira geral, pontuação mais elevadas são indicativas de posturas positivas por parte dos profissionais. Estudos anteriores de validação desse instrumento afirmam bons parâmetros psicométricos observados em bons índices de confiabilidade e consistência interna (Vargas \& Rocha, 2016; Vargas \& Luis, 2008).

A análise da associação entre variáveis sociodemográficas e as pontuações da escala EAFAAA foi realizada ajustando modelos de regressão linear múltipla com resposta normal após análise univariada de cada variável sociodemográfica 
independentemente. Nos modelos de regressão linear múltipla foram inseridas as variáveis com $p<0,20$ na análise univariada. No modelo final, as associações foram consideradas estatisticamente significativas se $p<0,05$ (Polit \& Beck, 2018). Os dados foram analisados por meio do software Statistical Package for the Social Sciences- versão 21.0.

Em consideração aos procedimentos éticos (Ministério da Saúde [MS], 2012), a pesquisa foi apreciada pelo Comitê de Ética em Pesquisa da Faculdade de Medicina de Botucatu recebendo sua aprovação em seis de novembro de 2017, por meio do Parecer Consubstanciado n. ${ }^{\circ}$ 2.367.217 e CAAE: 77941417.9.0000.5411.

\section{Resultados}

O perfil sociodemográfico da amostra caracterizou-se pelo predomínio de trabalhadores do sexo feminino, da cor branca, na faixa etária entre 20 e 35 anos, solteiros, com pós-graduação lato sensu completa fora da especialidade de atuação e a maioria da equipe de Enfermagem (nível médio e superior). As demais variáveis estão apresentadas na Tabela 1. 
Research, Society and Development, v. 10, n. 9, e11910917774, 2021

(CC BY 4.0) | ISSN 2525-3409 | DOI: http://dx.doi.org/10.33448/rsd-v10i9.17774

Tabela 1 - Perfil sociodemográfico da amostra de estudo. (n=42). Brasil. 2018.

\begin{tabular}{|c|c|c|c|}
\hline \multicolumn{2}{|c|}{ Variáveis } & \multirow{2}{*}{$\begin{array}{ll}\mathbf{n} \\
36\end{array}$} & \multirow{2}{*}{$\begin{array}{l}\% \\
85,7\end{array}$} \\
\hline Soxo & Feminino & & \\
\hline Sexo & Masculino & 6 & 14,3 \\
\hline \multirow{2}{*}{ Etnia } & Branca & 34 & 81,0 \\
\hline & Pardo & 8 & 19,0 \\
\hline \multirow{3}{*}{ Faixa etária (anos) } & 20 a 35 & 17 & 40,5 \\
\hline & 36 a 50 & 16 & 38,1 \\
\hline & $\geq 51$ & 9 & 21,4 \\
\hline \multirow{4}{*}{ Estado civil } & Solteiro & 19 & 45,2 \\
\hline & Com companheiro & 16 & 38,1 \\
\hline & Divorciado & 6 & 14,3 \\
\hline & Viúvo & 1 & 2,4 \\
\hline \multirow{5}{*}{ Nível de Escolaridade } & Técnico completo & 11 & 26,2 \\
\hline & Superior completo & 10 & 23,8 \\
\hline & Superior incompleto & 1 & 2,4 \\
\hline & Pós graduação completa & 18 & 42,9 \\
\hline & Pós graduação incompleta & 2 & 4,7 \\
\hline \multirow{4}{*}{ Área de Especialização } & Lato sensu em saúde mental & 6 & 24,0 \\
\hline & Lato sensu fora da área & 15 & 60,0 \\
\hline & Mestrado fora da área & 2 & 8,0 \\
\hline & Doutorado fora da área & 2 & 8,0 \\
\hline \multirow{4}{*}{$\begin{array}{l}\text { Tempo de trabalho na saúde } \\
\text { mental (anos) }\end{array}$} & 1 & 3 & 7,15 \\
\hline & 2 e 4 & 21 & 50,0 \\
\hline & 5 e 7 & 14 & 33,3 \\
\hline & $\geq 8 \mathrm{~s}$ & 4 & 9,55 \\
\hline \multirow{3}{*}{$\begin{array}{l}\text { Tempo de trabalho no serviço } \\
\text { (anos) }\end{array}$} & $\overline{1}$ & 4 & 9,5 \\
\hline & 2 e 4 & 23 & 54,8 \\
\hline & 5 e 7 & 15 & 35,7 \\
\hline \multirow{8}{*}{ Profissão } & Médico & 1 & 2,3 \\
\hline & Psicólogo & 3 & 7,1 \\
\hline & Técnico/auxiliar de enfermagem & 13 & 31,1 \\
\hline & Terapeuta ocupacional & 2 & 4,7 \\
\hline & Enfermeiro & 13 & 31,1 \\
\hline & Assistente Social & 5 & 11,9 \\
\hline & Educador físico & 1 & 2,3 \\
\hline & Outros & 4 & 9,50 \\
\hline \multirow{3}{*}{$\begin{array}{l}\text { Carga Horária Semanal } \\
\text { (horas) }\end{array}$} & 20 & 1 & 2,4 \\
\hline & 30 & 32 & 76,2 \\
\hline & 40 & 9 & 21,4 \\
\hline \multirow{2}{*}{ Vínculo empregatício } & Celetista & 38 & 90,5 \\
\hline & Estatutário & 4 & 9,5 \\
\hline
\end{tabular}

Fonte: Autores.

A Tabela 2 ilustra os escores, global e por fatores que compõem a EAFAA, obtidos pelos participantes dessa pesquisa. Segundo a pontuação global, pode-se inferir uma tendência para atitudes positivas por parte dos profissionais frente ao álcool, alcoolista e alcoolismo. Os fatores 3 e 4 que correspondem, respectivamente, à etiologia e ao uso de álcool indicam tendências negativas. Tendências positivas foram observadas nos dois primeiros fatores que abordam o trabalhar e relacionar-se com o alcoolista (Fator 1) e atitudes frente ao alcoolismo (Fator 2). 
Tabela 2 - Atitudes dos trabalhadores de um serviço de referência em álcool e outras drogas frente ao álcool, alcoolista e alcoolismo $(n=42)$. Brasil, 2018.

\begin{tabular}{lcccc}
\hline EAFAAA & Média (DP) & Mediana & Escore mínimo & Escore máximo \\
\hline Fator 1 & $3,7(0,5)$ & 3,8 & 2,4 & 4,7 \\
Fator 2 & $3,7(0,8)$ & 3,6 & 2,2 & 5,0 \\
Fator 3 & $2,7(0,4)$ & 2,7 & 2,0 & 3,5 \\
Fator 4 & $2,8(0,6)$ & 2,9 & 1,3 & 4,2 \\
Global & $3,3(0,4)$ & 3,4 & 2,5 & 4,0 \\
\hline
\end{tabular}

Fonte: Autores.

O modelo de regressão linear múltipla ajustado para cada fator da EAFAAA está apresentado na Tabela 3. Enfatiza-se que na análise univariada, as variáveis independentes, sexo, etnia, estado civil e vínculo empregatício não apresentaram fortes associações ( $\mathrm{p}<0,20)$, de modo que não foram elegíveis para o modelo ajustado. A mesma ocorrência se deu com o desfecho da EAFAAA - Fator 3 que não apresentou forte associação com nenhuma variável independente.

Como resultado, as variáveis sociodemográficas e os escores da EAFAAA separados por fatores ou global não apresentaram associações que explicassem os resultados obtidos nas atitudes dos profissionais frente ao álcool, alcoolista e alcoolismo.

Tabela 3 - Modelo de regressão linear múltipla ajustado para cada fator da EAFAAA (n=42). Brasil. 2018.

\begin{tabular}{|c|c|c|c|c|c|c|c|c|}
\hline \multirow[b]{3}{*}{ Variáveis } & \multicolumn{8}{|c|}{ Desfechos } \\
\hline & \multicolumn{2}{|c|}{ Global } & \multicolumn{2}{|c|}{ Fator 1} & \multicolumn{2}{|l|}{ Fator 2} & \multicolumn{2}{|l|}{ Fator 4} \\
\hline & $\beta(\mathrm{IC})$ & $\mathrm{p}$ & $\beta(\mathrm{IC})$ & $\mathrm{p}$ & $\beta(\mathrm{IC})$ & $\mathrm{p}$ & $\beta(\mathrm{IC})$ & $\mathrm{p}$ \\
\hline Idade (anos) & - & - & - & - & $\begin{array}{c}-0,05 \\
(-0,31-0,22)\end{array}$ &, 73 & $\begin{array}{c}-0,16 \\
(-0,222-0,19)\end{array}$ & ,87 \\
\hline $\begin{array}{l}\text { Escolaridade } \\
\quad(\text { anos })\end{array}$ & $\begin{array}{c}3,301 \\
(-1,078-7,68)\end{array}$ & ,13 & $\begin{array}{c}1,2 \\
(-1,5-3,8)\end{array}$ &, 37 & $\begin{array}{c}1,02 \\
(-1,06-3,11)\end{array}$ &, 33 & $\begin{array}{c}1,007 \\
(-0,643-2,66)\end{array}$ & ,22 \\
\hline $\begin{array}{c}\text { Pós Graduação na } \\
\text { área }\end{array}$ & $\begin{array}{c}12,336 \\
(-10,42-35,09)\end{array}$ &, 27 & $\begin{array}{c}9,3 \\
(-3,7-22,4)\end{array}$ &, 15 & $\begin{array}{c}7,20 \\
(-2,60-17,01)\end{array}$ &, 14 & $\begin{array}{c}1,029 \\
(-6,194-8,25)\end{array}$ &, 77 \\
\hline $\begin{array}{c}\text { Pós Graduação } \\
\text { fora da área }\end{array}$ & $\begin{array}{c}0,733 \\
(-19,55-21,01)\end{array}$ & ,94 & $\begin{array}{c}-2,3 \\
(-13,9-9,3)\end{array}$ & ,69 & $\begin{array}{c}-2,18 \\
(-10,93-6,57)\end{array}$ & ,62 & $\begin{array}{c}-0,390 \\
(-6,765-5,99)\end{array}$ & ,90 \\
\hline $\begin{array}{l}\text { Tempo de atuação } \\
\text { em saúde mental }\end{array}$ & - & - & - & - & - & - & $\begin{array}{c}-0,838 \\
(-7,519-5,84)\end{array}$ &, 80 \\
\hline $\begin{array}{c}\text { Tempo de atuação } \\
\text { no serviço }\end{array}$ & - & - & - & - & - & - & $\begin{array}{c}-2,172 \\
(-9,182-4,84)\end{array}$ &, 53 \\
\hline
\end{tabular}

Fonte: Autores.

\section{Discussão}

Os resultados evidenciaram as atitudes dos trabalhadores de um serviço especializado no atendimento a pessoas com transtornos relacionados ao uso de substâncias. Nessa população, observou-se tendência para atitudes positivas, com destaque para as dimensões teóricas referentes ao trabalho e à relação estabelecida com o alcoolista, bem como, à pessoa considerando suas características físicas e psíquicas.

Os fatores referentes à etiologia e ao uso do álcool evidenciaram tendências para atitudes negativas por parte dos trabalhadores que correspondem, na dimensão micropolítica, à influência do paradigma proibicionista (Gomes-Medeiros, Farias, Campos \& Tófoli, 2019) sobre as concepções dos profissionais que sustentam os respectivos resultados. Desse modo, 
os dados dessa investigação indicam a importância de ações no campo do cuidado e da formação que contribuam para o desenvolvimento do modo de atenção psicossocial para o cuidado à pessoa com transtornos decorrente do uso de álcool.

Diferentemente dos resultados obtidos nessa pesquisa, uma amostra de enfermeiros colombianos que trabalhavam, predominantemente, em serviços hospitalares generalizados apresentou tendência para atitudes negativas ou ambivalentes considerando, inclusive, os mesmos fatores que se mostraram positivos nesse estudo, isto é, o trabalho, a relação e a pessoa (Ramírez, Vargas \& Luis, 2019). Ao investigar trabalhadores de Centros de Atenção Psicossocial Álcool e Outras Drogas no município de São Paulo, observou-se resultados semelhantes ao encontrado nessa investigação (Pinho et al., 2018; Barbosa, Cobayashi \& Oliveira, 2017), ou seja, o escore geral detectou tendência de atitudes positivas, em especial, para os fatores trabalho, relação e etiologia.

Outra pesquisa realizada em Minas Gerais - Brasil com 50 enfermeiros atuantes em Estratégia Saúde da Família (ESF) apontou que a maioria dos enfermeiros apresenta tendência a atitudes negativas frente ao álcool, ao alcoolismo e ao alcoolista. Afirma que em relação ao conhecimento a respeito do álcool e do alcoolismo, os enfermeiros mostram fragilidades em reconhecer os problemas relacionados ao uso do álcool (Bezerra, Freitas \& Amendola, 2020).

Importa ressaltar as distintas características entre as populações, uma vez que essa pesquisa investigou uma equipe multiprofissionais de trabalhadores que atuam em um serviço hospitalar especializado. Nessa direção, corrobora o fato de que profissionais com contato frequente com alcoolista desenvolvem atitudes positivas em relação ao trabalho e à relação (Ramírez, Vargas \& Luis, 2019), conforme apontam as dimensões teóricas dessa investigação.

Ao aplicar a EAFAAA em profissionais da Atenção Primária à Saúde, identificou-se tendência de atitudes positivas frente às características da pessoa com transtornos decorrentes do uso de álcool (Caixeta, Pedrosa, \& Haas, 2016) e atitudes negativas referentes ao trabalho e à relação estabelecida (Caixeta, Pedrosa \& Haas, 2018), resultados distintos aos encontrados na população desse estudo. Além disso, profissionais do sexo masculino e sem parceiros foram duas características sociodemográficas que estiveram associadas a desfechos positivos em relação às atitudes, e a formação específica na área não representou uma variável que influenciou nas atitudes demonstradas pelos trabalhadores (Caixeta, Pedrosa \& Haas, 2018).

Similar ao contato frequente, a formação específica para o atendimento da pessoa com transtorno decorrente do uso de álcool pode influenciar no desenvolvimento de atitudes positivas (Pinho et al., 2018; Ramírez, Vargas \& Luis, 2019; Soares, Vargas \& Formigoni, 2013). Contudo, segundo os resultados do presente estudo, não se apontou associação entre pósgraduação e atitudes frente dos trabalhadores frente às diferentes dimensões teóricas avaliadas na escala. Indica-se como possível explicação, o fato de que dentre aqueles participantes que declararam pós-graduação, $76 \%$ realizaram em áreas distintas da saúde mental ou álcool e outras drogas.

O desenvolvimento de habilidades para desempenhar o cuidado junto a pessoas com transtornos relacionados ao uso de álcool tem relação com aspectos da graduação. Nos primeiros anos da graduação de enfermagem, observou-se um conjunto de afetos ambivalentes mobilizados no estudante durante o atendimento simulado a uma pessoa com uso nocivo de álcool e mesmo desempenhando comportamento ético e tendência para o cuidado integral, notou-se concepções reducionistas por parte dos estudantes, como, imprevisibilidade e periculosidade (Rodrigues \& Souza, 2018). Quando no término da formação, os estudantes afirmaram que há necessidade de maior ênfase teórica e prática acerca do uso nocivo de álcool, considerando que o aprendizado não foi efetivo para a demanda e para a reconstrução de suas crenças (Tavares, Reinaldo \& Silveira, 2017).

Outro contexto pode ser observado na graduação de medicina, em uma amostra de escolas do sudeste brasileiro observou-se que do segundo ao quinto ano há um aumento dos conhecimentos e melhora na qualificação das atitudes dos estudantes em relação aos transtornos decorrentes do uso de álcool. Não foi possível identificar as modificações acerca das atitudes detalhadamente, mas os resultados indicaram que atitudes mais positivas estão associadas ao fato de o estudante não adotar padrão de consumo episódico (Aguiar, Castelli, Toledo, Ubaldo, Silva \& Fonseca, 2018). 
Deve-se atentar para o fato de que os domínios teóricos etiologia e o uso de álcool ao indicarem tendências negativas denunciam uma postura fundamentada em uma concepção moralista que, por sua vez, reforça uma fragilidade da graduação em atuar sobre crenças e preconceitos dos estudantes. Possibilitar, durante a formação dos profissionais de saúde, espaços para a aprendizagem de conhecimentos, habilidades e atitudes para o cuidado à pessoa com transtornos decorrentes do uso de álcool representa um nó crítico (Rodrigues \& Souza, 2018; Tavares, Reinaldo \& Silveira, 2017; Aguiar et al., 2018; Muzyk et al., 2019).

Para tanto, tem-se discutido para os cursos de graduação a ampliação de abordagens teóricas e práticas sobre estratégias de redução de danos e tratamento medicamentoso, associar as substâncias responsáveis pela maior morbimortalidade desenvolvendo o reconhecimento de padrões de uso, possibilitar diversas aproximações durante a formação incluindo substâncias não tradicionalmente abordadas, utilizando estratégias de ensino-aprendizagem em primeira pessoa no exercício da interprofissionalidade (Muzyk et al., 2019).

Diferentes núcleos profissionais demonstram distintas atitudes na abordagem à pessoa com transtornos relacionados ao uso de substâncias no contexto da estratégia saúde da família. Observou-se que, entre uma amostra constituída por médicos, enfermeiros e dentistas, os primeiros demonstraram atitudes e práticas mais positivas, observadas na identificação dos usuários no território e manejo clínico. Em comum às três categorias profissionais, notou-se que quanto menor o tempo de trabalho e faixa etária, menor o conhecimento sobre o território de adscrição, dificultando o desenvolvimento de ações voltadas para o cuidado dessa população (Farias, Bernardino, Madruga, d’Ávila \& Lucas, 2019). Distintamente, na amostra dessa pesquisa não houve associações entre as variáveis profissionais e, as atitudes e as práticas clínicas desenvolvidas para a pessoa com transtornos decorrentes do uso de substâncias.

O delineamento transversal e a coleta de dados ter sido realizado em um determinado serviço de saúde correspondem às limitações dessa pesquisa.

\section{Conclusão}

As atitudes dos profissionais de saúde vinculados a um serviço de referência em álcool e outras drogas observaram na tendência para atitudes positivas nas dimensões teóricas referentes à relação e ao trabalho junto à pessoa com transtornos decorrentes ao uso de álcool, concomitantemente, atitudes negativas acerca das dimensões etiológicas e ao consumo dessa substância. Esses resultados não foram explicados pelas variáveis sociodemográficas da população de estudo, uma vez que não houve associações destas com o desfecho atitudes profissionais.

\section{Referências}

Aguiar, A. S., Catelli, R., Toledo, L., Ubaldo, L., Silva, C. J., \& Fonseca, V. A. S. (2018). Estudo da atitude diante do paciente alcoolista e do conhecimento sobre alcoolismo em função do padrão de beber de estudantes de medicina. Revista Brasileira de Educação Médica, 42(3), 49-56. http://dx.doi.org/10.1590/1981-52712015v42n3RB20180052

Barbosa, G. C., Cobayashi, K., \& Oliveira, M. A. (2017). Atitudes dos trabalhadores de um Centro de Atenção Psicossocial frente ao uso do álcool e alcoolismo. Revista Eletrônica de Graduação do UNIVEM, 10(1), 1-12. https://revista.univem.edu.br/REGRAD/article/view/1842

Bezerra, M. E. T., Freitas, N. O., \& Amendola, F. (2020). Álcool, alcoolismo e alcoolista: atitudes dos enfermeiros da estratégia saúde da família. Enfermagem em Foco, 11(3), 114-121. Acesso em 4 de abril de 2021 http://revista.cofen.gov.br/index.php/enfermagem/article/view/2789/897

Caixeta, L. M. M., Pedrosa, L. A. K., \& Haas, V. J. (2016). Analysis of attitudes of Primary Health Care professionals regarding people with disorders due to alcohol use. Revista Eletrônica Saúde Mental Álcool e Drogas, 12(2), 84-91. http://dx.doi.org/10.11606/issn.1806-6976.v12i2p84-91

Caixeta, L. M. M., Pedrosa, L. A. K., \& Haas, V. J. (2018). The work and the interpersonal relationships with patients presenting alcohol-related disorders. International Journal of Development Research, 8(7), 21907-13. https://www.journalijdr.com/work-and-interpersonal-relationships-patients-presentingalcohol-related-disorders Acesso em 4 de abril de 2021

Carvalho, A. F., Heilig, M., Perez, A., Probst, C., \& Rehm, J. (2019). Alcohol use disorders. Lancet, 394(10200), 781-792. http://dx.doi.org/10.1016/S01406736(19)31775-1 
Costa, P. H. A., \& Paiva, F. S. (2016). Revisão da literatura sobre as concepções dos profissionais de saúde sobre o uso de drogas no Brasil: modelo biomédico, naturalizações e moralismos. Physis: revista de saúde coletiva, 26(3), 1009-1031. http://dx.doi.org/10.1590/s0103-73312016000300015

Deng, Y., Wang, A. L., Frasso, R., Ran, M. S., Zhang, T. M., Kong, D., \& Wong, Y-L. I. (2021). Mental health-related stigma and attitudes toward patient care among providers of mental health services in a rural Chinese country. The International Journal of Social Psychiatry, 20764021992807. https://doi.org/10.1177/0020764021992807

Farias, L., Bernardino, I. M., Madruga, C. R. C., d`Ávila, S., \& Lucas, R. S. C. C. (2019). Atitudes e práticas de profissionais atuantes na Estratégia Saúde da Família quanto à abordagem aos usuários de drogas no município de Campina Grande, Paraíba, Brasil. Ciência \& Saúde Coletiva, 24(10), 3867-3878. http://dx.doi.org/10.1590/1413-812320182410.16772017

Gomes-Medeiros, D., Farias, P. H., Campos, G. W. S., \& Tófoli, L. F. (2019). Drug policy and Collective Health: necessary dialogues. Cadernos de Saúde Pública, 35(7), e00242618. http://dx.doi.org/10.1590/0102-311x00242618

Mangueira, S. O., Guimarães, F. J., Mangueira, J. O., Fernandes, A. F. C., \& Lopes, M. V. O. (2015). Promoção da saúde e políticas públicas do álcool no Brasil: revisão integrativa de literatura. Psicologia e Sociedade, 27(1), 157-168. http://dx.doi.org/10.1590/1807-03102015v27n1p157

Ministério da Saúde (2021). Conselho Nacional de Saúde. Resolução 466 de 12 de dezembro de 2012. Aprova as normas regulamentadoras de pesquisas envolvendo seres humanos. Brasília: Ministério da Saúde. http://conselho.saude.gov.br/resolucoes/2012/Reso466.pdf

Muzyk, A., Smothers, Z. P. W., Andolsek, K. M., Bradner, M., Bratberg, J. P., Clark, S. A., Collins, K., et al. (2019). Interprofessional substance use disorder education in health professions education programs: a scoping review. Academic Medicine: Journal of the Association of American Medical Colleges, 95(3), 470-480. http://dx.doi.org/10.1097/ACM.0000000000003053

Orsolini, L., Rojnić Palavra, I., Papanti, G. D., Potocan, M., Quattrone, D., Martens, M., \& Sklenářová, S., et al. (2021). Psychiatry Trainees' Attitudes, Knowledge, and Training in Addiction Psychiatry - A European Survey. Frontiers in Psychiatry, 11, 585607. http://dx.doi.org/10.3389/fpsyt.2020.585607

Pinho, P. H., Oliveira, M. A. F., Claro, H. G., Soares, R. H., Gonçalves, R. M. D. A., \& Pereira, M. A. (2018). Atitudes das equipes dos serviços de atenção psicossocial em álcool e drogas. Psicologia em Pesquisa, 12(1),1-10. http://dx.doi.org/10.24879/201800120010078

Polit, D. F., \& Beck, C. T. (2018). Fundamentos de Pesquisa em Enfermagem: avaliação de evidências para a prática de Enfermagem (9a ed.). Artmed.

Ramírez, E. G. L., \& Vargas, D de. (2017). Scale of attitudes toward alcohol - Spanish version: evidences of validity and reliability. Revista Latino-Americana de Enfermagem, 25, e2918. http://dx.doi.org/10.1590/1518-8345.1721.2918

Ramírez, E.G. L., Vargas, D. de, \& Luis, M. V. (2019). Atitudes frente ao álcool, ao alcoolismo e à pessoa com transtornos relacionados ao uso de álcool em enfermeiros colombiano. Cogitare Enfermagem, 24, e58795. http://dx.doi.org/10.5380/ce.v24i0.58795.

Richards, S. N., Broadus, A. D., \& Yerdelman, L. A. (2021). Treatment and non-nreatment professionals in Texas: race, sex, age, and level of education influencing attitudes about addiction. Journal of Drug Issues, 51(1), 218-235. http://dx.doi.org/10.1177/0022042620971857

Rodrigues, S. B., \& Souza, J. (2018). Atitudes de estudantes iniciantes da graduação em enfermagem diante do paciente alcoolista. Revista Eletrônica Saúde Mental Álcool e Drogas, 14(1), 37-44. http://dx.doi.org/10.11606/issn.1806-6976.smad.2018.000405

Soares, J., Vargas, D. de, \& Formigoni, M. L. O. S. (2013). Knowledge and attitudes of nurses towards alcohol and related problems: the impact of an educational intervention. Revista da Escola de Enfermagem da USP, 47(5), 1172-9. http://dx.doi.org/10.1590/S0080-623420130000500023

Tavares, M. L. O., Reinaldo, A. M. S., \& Silveira, B. V. (2017). Dimensões teórico-práticas na formação do enfermeiro: crenças e atitudes relacionadas ao alcoolismo. Revista Eletrônica Saúde Mental Álcool e Drogas, 13(3), 148-155. http://dx.doi.org/10.11606/issn.1806-6976.v13i3p148-155

Vargas, D. de, Bittencourt, M. N., Rocha, F. M., \& Silva, A. C. O. (2014). Alcohol/drugs community mental health services: insertion and practices of nursing professionals. Esc. Anna Nery, 18(1), 101-106. http://dx.doi.org/10.5935/1414-8145.20140015

Vargas, D. de, \& Luis, M. A. V. (2008). Development and validation of a scale of attitudes towards alcohol, alcoholism and alcoholics. Revista LatinoAmericana de Enfermagem, 16(5), 895-902. http://dx.doi.org/10.1590/S0104-11692008000500016

Vargas, D. de, \& Rocha, F. M. (2016). Psychometric properties of the Attitudes Scale facing Alcohol and Alcoholism in nursing students. Revista LatinoAmericana de Enfermagem, 24, e2823. http://dx.doi.org/10.1590/1518-8345.0774.2823.

Vistorte, A. O. R., Ribeiro, W. S., Jaen, D., Jorge, M. R., Evans-Lacko, S., \& Mari, J. J. (2018). Stigmatizing attitudes of primary care professionals towards people with mental disorders: A systematic review. The International Journal of Psychiatry in Medicine, 53(4), 317-338. https://doi.org/10.1177/0091217418778620

World Health Organization (2018). Global Status Report on Alcohol and Health. Switzerland: World Health Organization. Acesso em 4 de abril de 2021 https://www.who.int/substance_abuse/publications/global_alcohol_report/en/ 